\title{
Why am I always late? Modeling the cognitive mechanisms underlying anticipatory timing under uncertainty
}

\author{
Konstantina Sokratous \\ University of Florida \\ Alexander H. Wurm \\ University of Florida
}

\author{
Guy Hawkins \\ University of Newcastle \\ Peter D. Kvam \\ University of Florida
}

\begin{abstract}
Responding to stimuli in a timely manner and anticipating the timing of future events both require us to internally track the passage of time. Models of timing on these tasks suggest that the subjective passage of time can be described as a noisy accumulation process driven by neural oscillations. In this paper, we show that the accuracy of these accumulators can be manipulated by occluding visual cues to the passage of time. Using a simple perceptual paradigm, we manipulate the total length of time that a stimulus must be tracked, the rate at which it moves, and the uncertainty that participants have about its position (length of occlusion). Participants consistently under-estimated the movement of the stimulus when it was occluded, corresponding to a drift rate in an accumulator model that was approximately half of what would be required to accurately track the passage of time. This results in consistently tardy anticipatory response times under uncertainty (Study 1) and an under-estimation of stimulus movement as it passes behind an occlusion (Study 2). Using a novel timing problems scale, we show that individual differences in model parameters representing subjective tracking of time under uncertainty predicted real-world difficulties managing time, tardiness, and procrastination.
\end{abstract}

Keywords: anticipatory timing I evidence accumulation I response time | tardiness | procrastination

\section{Introduction}

Response times have long been used to understand the dynamic cognitive processes underlying perceptual decisionmaking and performance (Luce, 1986; MacLeod, 1992; Eriksen \& Eriksen, 1974). Looking at the entire distribution of response times elicited in response to a particular stimulus allows a researcher to make rich inferences about cognition that are not possible with simple measures of accuracy or central tendency (Balota \& Yap, 2011; Lerche \& Voss, 2020; Haines et al., 2020). Paired with models that account for distributions of response times (Ratcliff et al., 2016; Busemeyer et al., 2019), they can even reverse the conclusions that we draw about psychological processes relative to mean response times, by better characterizing the underlying psychological processes that generated the data (Heathcote et al., 1991; Algom et al., 2015). However, response times have historically focused on reactive responses or decisions, where a participant enters a response at the time of their choosing in response to a presented stimulus. Sometimes these reactive responses are constrained by a response deadline or other types of time pressure (Wickelgren, 1977; Reed, 1973; Edland \& Svenson, 1993; Heitz, 2014), but for participants, the time at which responses are entered is typically secondary to selection of a response itself. That is, the goal is to decide which option to select while minimizing response time (Wald \& Wolfowitz, 1949; Hawkins et al., 2012; Bogacz et al., 2006, 2010).

Although common, reactive decision-making is not the only type of task where timing is important. For example, imagine you are trying to trap or swat a fly that has come inside. Successfully doing so requires being able to predict precisely where the fly will be and when, integrating your knowledge of its trajectory to predict the best time to take action. The task is naturally made more difficult when the trajectory of the fly or its future location is more uncertain, either because you cannot follow its flight (e.g., it moves too fast) or because it becomes occluded (e.g., flies behind an item of furniture). In this scenario, you are not spontaneously reacting to some event, but rather you are engaging in anticipatory timing with the goal to calibrate your actions for an event you know is coming (Balc1 \& Simen, 2016). This type of task can be juxtaposed against traditional response time tasks. As opposed to merely assessing latency, we can also measure accuracy and precision: how close were you to the correct timing of your action to catch the fly?

Anticipatory decision making paradigms are most common in the context of rhythmic motor production, where participants must tap along with or reproduce a beat of a specific 
frequency (Wing \& Kristofferson, 1973b,a; Michon, 1967; Ivry, 1996). These often mimic the types of decisions and actions performed by musicians, who make hundreds or even thousands of precisely-timed actions over the course of a single piece of music. In motor production and timing tasks, a participant must make a decision about when to respond and prepare for execution of a single action, rather than selecting among multiple different responses to make. Performance on these tasks is gauged in terms of the difference between the correct response time (e.g., when the beat actually occurs) and the time at which a participant responds.

Timing deficits on these motor production and timing tasks are related to a myriad of mental health and neurological disorders including bipolar disorder (Carroll et al., 2009), schizophrenia (O'Donnell et al., 2004), Tourette's (Martino et al., 2019), ADHD (Zelaznik et al., 2012), dyslexia (Wolff, 2002; Casini et al., 2018), depression (Lemke et al., 1999; Williamson et al., 2014), substance use (Wittmann et al., 2007), and of course Parkinson's and related neurocognitive disorders (C. R. G. Jones et al., 2011; C. R. Jones \& Jahanshahi, 2014; Bareš et al., 2010). Modeling the cognitive mechanisms underlying anticipatory timing can help diagnose and understand motor deficits, providing insight into clinical disorders that are difficult or impossible to infer from behavior alone (Hitchcock et al., 2021). Interestingly, performance on low-level motor production and timing tasks appears to be related to both time perception (Ivry, 1996) and to relatively "distant" tasks such as high-level planning (Ziessler \& Nattkemper, 2011), time management, and expert performance on spatio-temporal tasks (Keele \& Hawkins, 1982). Training on time perception tasks even appears to generate lasting improvements in motor production (Meegan et al., 2000) and conversely, motor training can yield improvements in time perception and temporal expectations (Fautrelle et al., 2015).

The wide-ranging correlates of simple timing abilities suggest that the basic cognitive mechanisms underlying timing ability subserve complex behavior across multiple domains. However, it is not always clear which components of timing ability are responsible for performance on different tasks. In this paper, we examine response times on an anticipatory response task and use a timing model (Anders et al., 2016; Balc1 \& Simen, 2016) to disentangle cognitive processes related to tracking the passage of time, representing uncertainty in the timing of future events, and triggering actions at a particular time. We then examine how these functions (or dysfunctions) propagate to high-level time management, connecting model parameters to procrastination selfreport scales and a new scale measuring generalized problems with lateness, planning, and time management.

One might expect that anticipatory response times leverage cognitive mechanisms different from those used to produce decisions in reactive response time paradigms, due to the distinct nature of the tasks between the two concepts. However, recent work by Hawkins \& Heathcote (2021) has suggested that common reactive decision tasks may be controlled in part by a timer mechanism, where a participant's internal sense of time (leveraged in anticipatory tasks) is used to impose deadlines on the evidence accumulation process typically thought to control decision-making (Ratcliff et al., 2016; Busemeyer et al., 2019). In this view, a "timer" process runs in parallel to evidence accumulation and halts the decision process when it hits a threshold (target duration), forcing an early response based on whatever information the decision-maker has available at that time. Introducing such a mechanism can be quite useful, as keeping track of time during choice suggests that difficult decisions can be terminated early, and that a decision-maker can move onto easier - but equally important- choices.

In this sense, Hawkins \& Heathcote's (2021) timed racing diffusion model (TRDM) shares goals with models of collapsing choice boundaries (Bowman et al., 2012; Drugowitsch et al., 2012; Hawkins et al., 2015) as well as urgency signal / urgency-gating models (Churchland et al., 2008), each of which imposes a choice deadline by supplementing the evidence accumulation process with a timer mechanism, tracking the elapsed time since the beginning of the decision process. Naturally, this is an intersection where decision models should be informed by models of timing: how exactly do people keep track of time, and do these mechanisms resemble the ones that have been proposed to terminate evidence accumulation? Recent work on collapsing boundary models have suggested that timing ability is related to a general tendency towards response caution, but not to the rate of collapse of thresholds (Miletić \& van Maanen, 2019). Different plausible causes could account for this result, for instance separate cognitive mechanisms might control timing and decision-making.

Alternatively, it could be that collapsing boundaries do not reflect the true mechanisms underlying early termination of decision processes under time pressure. Connections between decision-making and timing are much stronger when both are viewed through the lens of diffusion processes (Simen et al., 2016; Ratcliff, 1978a), where our ability to accumulate information or track time is driven by integrating spikes produced by neural oscillators over time (Simen et al., 2011; Smith, 2010). Balc1 \& Simen (2016) developed a model of timing based on the same approach used by Hawkins \& Heathcote (2021): the duration of time that has passed since being exposed to a stimulus is tracked by a noisy accumulator; specifically, its level of activation corresponds to the number of "ticks" of a neural oscillator, thereby reflecting the approximate duration of time that has elapsed. This approach to modeling timing processes makes a number of predictions for how people must represent the passage of time as they perform a variety of perceptual tasks. In the 
next section, we review the structure of this model and the empirical predictions we can derive from it.

\section{Timing model}

In both the TRDM (Hawkins \& Heathcote, 2021) and the time-adaptive, opponent Poisson drift diffusion model (TopDDM; Balc1 \& Simen, 2016; Simen et al., 2011), the passage of time is tracked by the degree of activation of a noisy accumulator. A diagram of this approach is shown in Figure 1. As time passes, oscillatory neurons generate spikes of activity that are integrated into a timing accumulator $x$. This produces a continuous-time random walk / diffusion process (Ratcliff \& McKoon, 2008; Ratcliff et al., 2016), where the state of the accumulator over time changes according to a Wiener process $W_{t}$ with constant drift $\mu$ :

$$
d x=\mu \cdot d t+\sigma \cdot d B_{t}
$$

The state of the accumulator at a particular time $t$ is indexed by $x(t)$. If the process starts at position $x(0)=0$, then its state at time $t>0$ is normally distributed, with mean $\mu t$ and variance $\sigma^{2} t$. The variance is multiplied by a Brownian motion component, which results in a stochastic process with linearly increasing variability over time. Therefore, we expect that the drift rate $\mu$ in a diffusion framework describes the linear correspondence between the objective passage of time $(t)$ and the subjective representation of its passage $(x(t))$. Values of $\mu$ less than one (when the process is scaled correctly) correspond to cases where the decision maker perceives the passage of time to be slower than it is in actuality, whereas values greater than one correspond to cases where time subjectively appears to move more quickly. Therefore, we might expect the value of $\mu$ to be altered in cases of novel environments or stimuli (Pariyadath \& Eagleman, 2007; Ulrich et al., 2006), shifts in attention (Tse et al., 2004) or mood (Hoffer \& Osmond, 1962), stimulus contrast (Kim \& McAuley, 2013), or even aging (Wittmann \& Lehnhoff, 2005). In this paper, we evaluate the model's ability to accommodate changes in subjective time by testing how $\mu-$ as well as each of the other model parameters - responds to stimulus manipulations of speed, uncertainty, and exposure.

Just as the drift rate corresponds to the average subjective passage of time, the value of the diffusion rate $\sigma^{2}$ reflects the relative uncertainty in the accumulator's state, describing how precisely a decision-maker tracks the passage of time. Greater values of $\sigma$ correspond to greater accumulation of momentary uncertainty, reflecting the integration of an increasing number of noisy neural signals generated by neural oscillators (Wing \& Kristofferson, 1973b; Balc1 \& Simen, 2016). Smaller values indicate a more precise subjective representation of time, although this representation can still be inaccurate if $\mu$ is not close to one (i.e., a biased representation of time, with low variance).
This subjective representation of time is mapped onto an anticipatory response by comparing the value of $x(t)$ to a threshold, $\theta$, as shown in Figure 1. Typically, there is a single threshold assigned to each response in reactive choice tasks (Vickers, 1970; Ratcliff et al., 2016) (though see Smith, 2016; Reynolds et al., 2021, 2020, for some exceptions), and a response is generated when $x(t)$ crosses one of the thresholds for the first time. In the case of timing, often there is only one response required and thus only one threshold.

In reactive choice models, the total response time is the sum of the time to hit this threshold, plus the time required to execute the response or non-decision time, $\tau$, forming a (shifted) Wald distribution (Anders et al., 2016). This nondecision time is sometimes abbreviated as $T_{E R}$, or the time for Encoding and Retrieval processes unrelated to the time taken to make the decision itself. However, the meaning of the shift produced by the non-decision time changes in anticipatory response times. In these tasks, a participant should already have encoded the stimulus and prepared their motor response by the time they expect to trigger a response. The "true" non-decision time should be approximately $\tau=0$. Therefore, a fixed delay relative to the true time at which participants should respond in anticipatory timing tasks $(\tau>0)$ instead reflects a failure on the participant's part to account for how long these processes take, creating a late response. Conversely, some participants may "jump the gun" and respond too early $(\tau<0)$, which is not possible with the conceptualization of non-decision time in classical reactive choice paradigms. When we examine the estimates of nondecision time later in the paper, we refer to $\tau$ in terms of biases toward early $(\tau<0)$ or late $(\tau>0)$ responses.

If a decision maker aims to respond at a target time $t$, then they will produce the best results when $\frac{\theta}{\mu}=t$ and when $\tau=0$. Due to the presence of noise in the representation of stimulus timing $(\sigma)$ there is unavoidable variability in response times around this point. But this point at least gives us a normative basis for performance on the task: participants with accurate perceptions of time on the task and good estimates of their own motor execution time should have parameters approximating these relationships.

\section{Model predictions}

The goal of this paper is to evaluate how the components of the aforementioned model of timing respond to manipulations of a moving stimulus, as well as the degree to which the model can account for patterns of response times (Study 1) and responses (Study 2) when attempting to track the passage of time. If participants' perceptions of time are controlled by a noisy accumulator, then we should observe corresponding patterns of behavior in anticipatory timing. We test these predictions using a moving-ball stimulus described in detail in the Methods. Subjects viewed the motion of the ball while it crossed their screen, before the same objected disappeared 


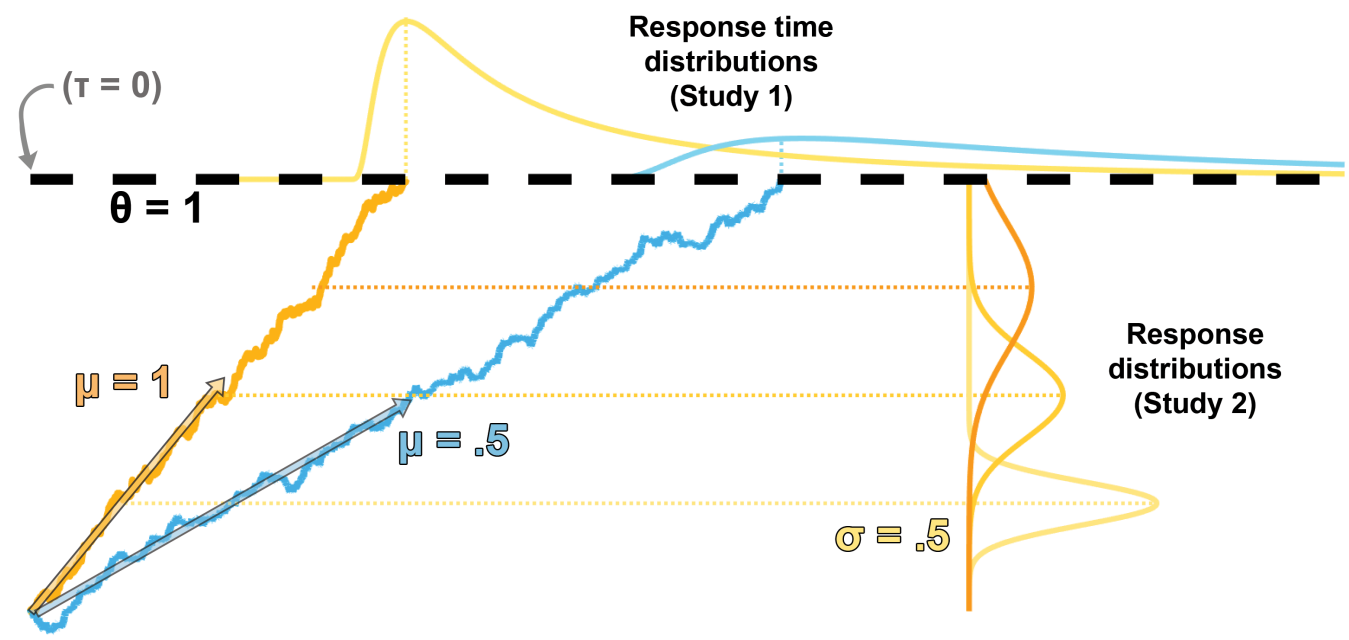

\section{Figure 1}

Diagram of the structure of the Wald model. In the first study, we examine distributions of observed response times (top), which are expected to follow a Wald distribution, and how they relate to manipulations of model parameters related to anticipatory timing. In the second study (right), we examine distributions of estimated stimulus locations, tapping into the evidence representations at particular points in time, which are expected to follow a normal distribution.

behind an occluded obstacle. At this point, it becomes vital to mentally represent the location of the now imperceptible ball as it continues on its trajectory, in order to predict the eventual arrival of the object at a specific position.

In this task, participants must assess the velocity of the ball to predict the timing of its movement through the occluded zone (Figure 2). In the first study, the objective was to predict when the ball will arrive at the far edge of the occlusion. The model predicts that the response times will follow a Wald distribution (Balcı \& Simen, 2016; Anders et al., 2016; Hawkins \& Heathcote, 2021). Furthermore, their uncertainty about its location should grow with the length of time the ball is occluded (Figure 1), as the variance of the accumulator representation grows linearly with time. We can also expect that the rate of accumulation $\mu$ will describe the ball's speed relative to the threshold $\theta$; therefore yielding higher values of $\mu$ for faster ball movements, when $\theta$ is fixed. Finally, the noise in accumulation (or variability in the rate; interchangeable in this paradigm) $\sigma$ could be affected by manipulations of the duration of exposure to the ball prior to occlusion; a greater exposure should yield a more accurate representation of the ball's speed, and thus more precise predictions for its arrival time.

In the second study, we test several additional predictions of the model by challenging participants to identify the location of the ball at a particular time. If the model predictions are borne out, an individual's prediction of its location at any given time should follow a normal distribution (truncated, as they know the ball could never be found outside the occlusion). Uncertainty about its eventual position should grow linearly with time, meaning that the variance of their location responses should correspond linearly with the length of time the ball remained occluded, before the prompt for its location (see right side of Figure 1). Manipulations of ball speed, length of occlusion, and exposure duration before the ball enters the occlusion should have the same effects on the parameters of the model in both Study 1 and Study 2 tasks.

Finally, we test two normative predictions of the model. First, an accurate timer should always track the location of the ball when its velocity has been properly perceived. Therefore, the mean value of $\frac{\theta}{\mu}$ for each participant should approach the exact amount of time it takes the ball to reach the far edge of the occlusion. Second, if participants appropriately prepare their motor responses and do not under- or over-estimate the time to execution, we should find that $\tau$ approaches the length of time for which the ball is shown on the screen prior to occlusion. Or instead, they should approach $\tau=0$ if we track the time between when the stimulus enters the occlusion and when participants enter their responses, rather than the length of time between the start of the trial and participants' responses.

These normative predictions allow us to decompose the cognitive mechanisms underlying timing ability, and in particular explain patterns of non-normative behavior. To preview our results, we find that almost all participants systematically make late responses relative to the true arrival time of the stimulus. Furthermore, the degree to which a participant gravitated toward late responding on these simple perceptual decisions was predictive of real-world difficulties with timing, such as patterns of lateness, difficulty with time manage- 
ment, and procrastination.

\section{Methods}

To examine patterns of anticipatory timing, we used a perceptual paradigm to expose participants to a moving ball and asked them to predict when the ball would reach a target location (Study 1) or where the ball would be at a given point in time (Study 2), both represented schematically in Figure 2. Partway along its trajectory, the ball was occluded, moving behind a gray box so that it was no longer visible. To challenge participants and explore how they responded to varying levels of uncertainty, we manipulated three aspects of the task: the speed at which the ball moved, the length of time the ball was occluded, and the distance the ball had to travel. Respectively, this allowed us to test the flexibility of participants' internal timers; the degree to which participants could represent uncertainty in the ball's position; and how quickly / reliably participants could assess its velocity for a given duration of exposure to the stimulus.

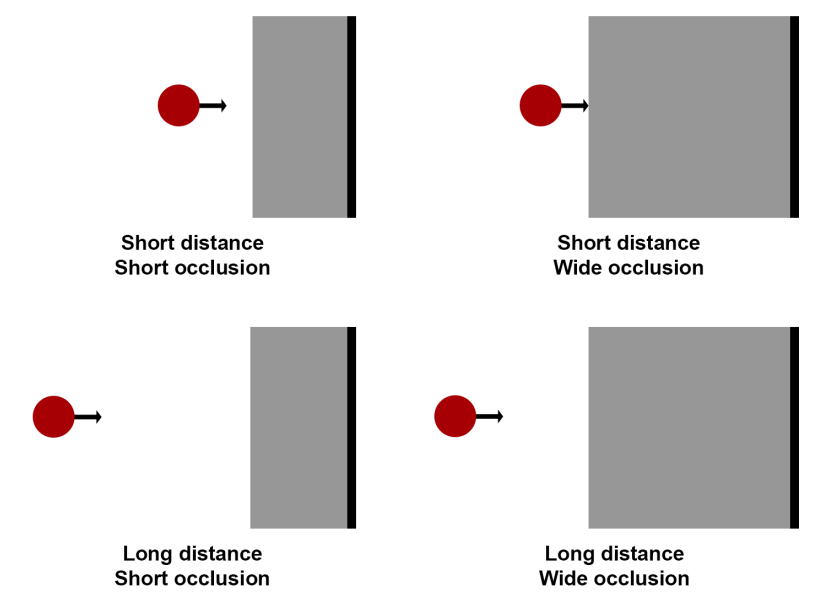

Figure 2

Diagram of four conditions of the task.

For simplicity, we denote the speed of the ball in different conditions as slow $(\mathrm{S})$ or fast $(\mathrm{F})$, the length of the occlusion as wide $(\mathrm{W})$ or narrow $(\mathrm{N})$, and the length of exposure to the ball before occlusion as short (S) or long (L). The combination of these manipulations resulted in 8 unique conditions $(2 \times 2 \times 2)$. In both experiments, each participant completed 320 trials grouped into 4 blocks of 80 trials, where each block included 10 trials from each of the 8 conditions. In total, they saw 40 trials of each unique combination of slow/fast ball speed, wide/narrow occlusion, and short/long distance. In Study 2, these 40 trials were further broken down into 4 location probes, described below.

In each study, there were multiple attention checks (such as "Select option $\mathrm{X}$ on the scale below before pressing continue") embedded in the directions for the perceptual task and self-report scales. Participants were removed from further analyses if they failed 2 or more of these checks or if their responses during the experiment fell outside of a reasonable range on more than $10 \%$ of trials. For Study 1, responses entered before the disappearance of the ball behind the occlusion were deemed unreasonable; while for Study 2, responses designating coordinates or an area outside of the occlusion were also deemed as such and removed from analysis. In both cases, participants were instructed as to what these ranges were. All subjects received US $\$ 10$ for participating in either study, each of which took approximately 4045 minutes to complete.

\section{Study 1}

Study 1 focused on the response time component of anticipatory timing, whilst connecting performance on the task to the parameters of an established model of response times (Anders et al., 2016; Balc1 \& Simen, 2016) to high-level problems with time management. The experiment in Study 1 asked participants to enter a response when the leading edge of the ball stimulus (Figure 2) reached the right edge of the gray occlusion. Note that Figure 2 shows a quite large ball for visibility, but the ball used in the actual experiment had a radius of only 5 pixels to minimize any differences in response time between participants who were using the ball's leading edge (i.e., following the directions) or who used its center or trailing edge instead.

Response times were recorded both from the onset of the stimulus - when participants clicked on the "Start" button to begin the ball movement at the start of each trial - to the time they clicked the mouse again to enter their response. We also calculated the time between when the ball fully disappeared behind the occlusion and when they entered their responses. As a result, we were able to evaluate and fit the model solely to the occluded time, thus gathering information about how well - or not - people represent positions of moving objects and anticipate their arrival.

There was a coding error for Study 1 where the WideShortFast condition presented a WideShortSlow stimulus, resulting in double the number of WhiteShortSlow trials and no WideShortFast trials. In the analyses (Results section), these were coded correctly (i.e., all WideShortFast were coded as WideShortSlow instead) so this did not affect the inferences we made in the effect-coded models.

\section{Participants \& Procedure}

For Study 1, subject recruitment took place on Prolific Academic, accepting everyone that passed the screening for sufficient fluency in English language and normal or corrected-to-normal vision. After completing informed consent on Qualtrics, the experiment was presented in JavaScript using jsPsych (De Leeuw, 2015), which produces response times that are similar in all respects to in-person experiments 
coded with software such as Psychtoolbox, except for an average $\sim 25 \mathrm{~ms}$ shift / delay in recorded response times (de Leeuw \& Motz, 2016; Reimers \& Stewart, 2015; Hilbig, 2016). Participants completed self-report scales on Qualtrics, randomly counterbalanced with the perceptual task.

A total of 94 participants took part in Study 1, with the goal of retaining approximately 85 participants after assuming $10 \%$ attrition with quality control checks. The target of 85 was identified as the required number to reliably detect correlations between measures (e.g., model parameters and self-report scale scores) of at least $r=.2$, while achieving Bayes factors for the null hypothesis $(r=0)$ of $B F_{01}>3$ in at least $80 \%$ of cases where the true relationship was null. This is effectively the Bayesian equivalent of $\alpha=.05 / \beta=.20$ for linear correlations between measures. Of the 94 participants, 16 were removed for failing attention checks on at least 3 occasions or for responding prematurely before the disappearance of the ball on $10 \%$ or more of trials. The remaining 78 participants were 30.91 years old on average (SD $=10.43$ years $), 64 \%$ female $/ 36 \%$ male, and primarily UK / EU residents (67\%), US residents $(28 \%)$ or South American residents (3\%, one each from Peru, Brazil, and Mexico; the final participant resided in India).

\section{Self-report measures}

In addition to the 320 trials of the anticipatory timing task, participants completed three self-report scales aimed at understanding the consequences of anticipatory timing ability. The first scale - introduced and validated for the first time in this study - aimed to assess whether and how much people struggled with timing in their day-to-day lives. It asked participants to rate the degree to which they agreed with statements such as "I am frequently late to events I plan to attend" and "I sometimes under-estimate how long tasks will take" on a 5-point scale from "Strongly disagree" to "Strongly agree". We seek to determine if, and to what degree, problems with simple perceptual anticipation tasks were representative of fundamental difficulties with timing. A complete list of the items and available answers are provided in Table $\mathrm{S} 1$. From the responses on this measure, we computed a simple summary score by adding the response to each question, with specific questions reverse-coded, where each response was assigned a value of 1-5. A higher value on the summary score indicated greater problems with timing.

In addition to the new timing scale, we also evaluated timing problems related to procrastination and time management using Steel's procrastination scale (Steel, 2010). As with the timing scale, we used simple summary scores to describe participants' responses on the procrastination scale. Finally, we hypothesized that participants' performance on timing tasks, and timing problems more generally, might be impacted by their general tendency to think and plan. We therefore included a need for cognition scale (Cacioppo \& Petty, 1982).
This last measure was ultimately not related to any interesting model parameters or measures of performance, so we do not discuss it further.

\section{Study 2}

While the predictions of timing models are typically evaluated with respect to response times, they also make predictions for how internal timers themselves should change with respect to time. The procedure in Study 2 was designed to evaluate this component of timing models by examining how uncertainty in the location of a moving object (the ball) evolves over time as participants track its location. Rather than looking at anticipated arrival times, we assessed instead where participants believed the ball was at specific points in time. Our objective was to shed light on the various components of anticipatory timing ability that might be responsible for the patterns of performance in Study 1, such as the tendency of participants to respond too late relative to the true arrival time. Prompting responses at specific times allowed us to probe the underlying representations of ball position and velocity, isolating the dynamics of the timer mechanism (Wing \& Kristofferson, 1973b) that supports anticipatory timing behavior. As we outlined in the introduction, timing models make very specific predictions for how responses on such a task should be distributed (Figure 1, right). If the TopDDM and TRDM are correct, the value of the timer that tracks the position of the ball should be normally distributed at any given point in time (truncated, according to the instructions that the ball should be behind the occlusion), and its variability should increase linearly with time.

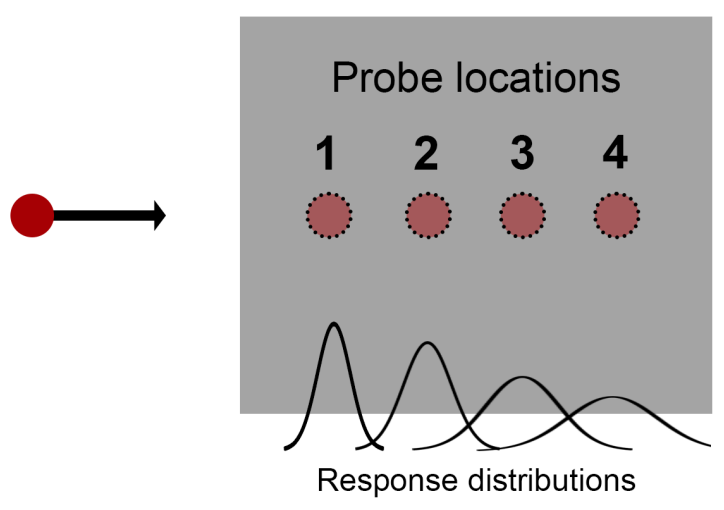

Figure 3

Diagram of the structure of the perceptual task in Study 2. The four probed locations (top) are expected to elicit linearly increasing variance in the response distributions (bottom) in each condition.

To test these predictions, Study 2 asked participants to estimate the location of the ball at a specifically prompted point in time. In addition to the eight conditions from Study 1 - 
with the same manipulations of ball speed, occlusion duration, and exposure duration - the location of the ball could be probed at any of four times after the ball had gone behind the occlusion. The probe was executed by the screen flashing black, followed by an inquiry as to the position of the center of the ball at the exact time the screen flashed. Within each condition, the flash could occur when the ball was $20 \%$, $40 \%, 60 \%$, or $80 \%$ of the way through the occlusion. This is illustrated in Figure 3. Following the probe, the $x$ and $y$ coordinates of the participant's next click on the screen were recorded as their response on that trial. After the click, the experiment would automatically advance to the next trial.

To calibrate the x-coordinate of participants' clicks, subjects were asked at the beginning of the experiment to click as precisely as they could on each of the edges of the gray square. This procedure was repeated twice, to ensure that we could measure as precisely as possible the location of participants' click relative to the sides of the occlusion. Once entered, the $\mathrm{x}$-location of each click was coded relative to these sides, from 0 (precisely at the left edge of the occlusion) to 1 (precisely at the right edge of the occlusion). Any responses outside this range were excluded from analysis; additionally, participants who responded outside the range on $10 \%$ or more of trials were excluded from further analysis altogether.

Normatively, we can derive even stronger predictions for performance on this type of task than the task in study 1 . A participant who is accurately representing the passage of time should be able to accurately identify the position of the ball as it moves behind the occlusion. In the model, this corresponds to a situation where the threshold divided by the drift rate is exactly proportional to the rate at which the ball is moving (the threshold:drift ratio and occlusion time are equal up to a constant). If participants are well calibrated to the position of the ball, then we should also find that estimates of $\tau$-which in the case of Study 2 will describe biases in the perceived location of the ball itself - are close to 0 .

\section{Participants \& Procedure}

For Study 2, participants were recruited from the University of Florida psychology participant pool. They participated in the experiment entirely online and received course credit for completing the study. As with the Prolific sample in Study 1, participants were permitted to participate as long as they indicated normal or corrected-to-normal vision and were fluent English speakers. They completed informed consent and self-report scales on Qualtrics, and the perceptual task was presented using JavaScript / jsPsych. Upon completing the study, participants were automatically redirected to the participant credit management system and granted credit for their participation.

A total of 30 participants completed the study. Of these, 8 participants failed attention checks or gave responses that were outside of the reasonable range (not within the gray box) on $20 \%$ or more of the trials. The remaining 22 participants were 19.5 years old on average ( $\mathrm{SD}=1.5$ years), including 64\% (14) female and 36\% (8) male participants. Participants were all U.S. residents, but identified most closely as $55 \%$ white, $32 \%$ Hispanic, $9 \%$ Asian, and $8 \%$ Black participants.

\section{Results}

All descriptive and generative model-based statistics / parameters were estimated using hierarchical Bayesian approaches, permitting us to look at both individual-level fits and group-level patterns in the data while allowing the two to impose mutual constraints (Kruschke, 2014; Lee \& Wagenmakers, 2014; Shiffrin et al., 2008). For descriptive models of basic behavioral outcomes (mean RTs, response locations), we use uninformative priors. For model-based analyses, we restrict the range of certain parameters based on their domain (e.g., thresholds and diffusion rates must be positive) but otherwise use weakly informative priors to guide the sampling process. However, priors on effects-coded model parameters were centered at zero and identical across all conditions, ensuring any observed parameter effects were driven by the data. For the exact specification of priors, see the code provided on the Open Science Framework (osf.io/b28sq).

For each descriptive and generative parameter, we report the mean of the posterior $(M)$ and the $95 \%$ highest-density interval (95\% HDI) that contains the $95 \%$ most likely values of the parameter based on the sampled posterior.

\section{Study 1}

For Study 1, response times were recorded as the time from stimulus onset to response. Naturally, these responses were comprised of both the time when the ball was behind the occlusion and the entire duration during which the ball was visible. We can expect response times recorded in JavaScript to be approximately $25 \mathrm{~ms}$ slower than the true response times (de Leeuw \& Motz, 2016), but this does not account for more than a trivial amount of the lateness of participants' responses. Judgments about subjects' timeliness - or lack thereof- were made while registering this particularity of the online format by subtracting $25 \mathrm{~ms}$ from all response times. Therefore, the online / JavaScript-dependent time shift no longer impeded measuring response times relative to normative values.

First, we can compare the overall patterns of response times to the time when they were supposed to be entered. These are shown in Figure 4. Overall, 77.96\% of responses were entered after the true arrival time of the ball, indicating a general tendency towards late responses. This suggests much more than trivial lateness, with responses trailing the true arrival time by approximately $661 \mathrm{~ms}$ out of a typical 15 s occlusion time $(M(R T)=0.66,95 \% \mathrm{HDI}=[0.64,0.68])$. 

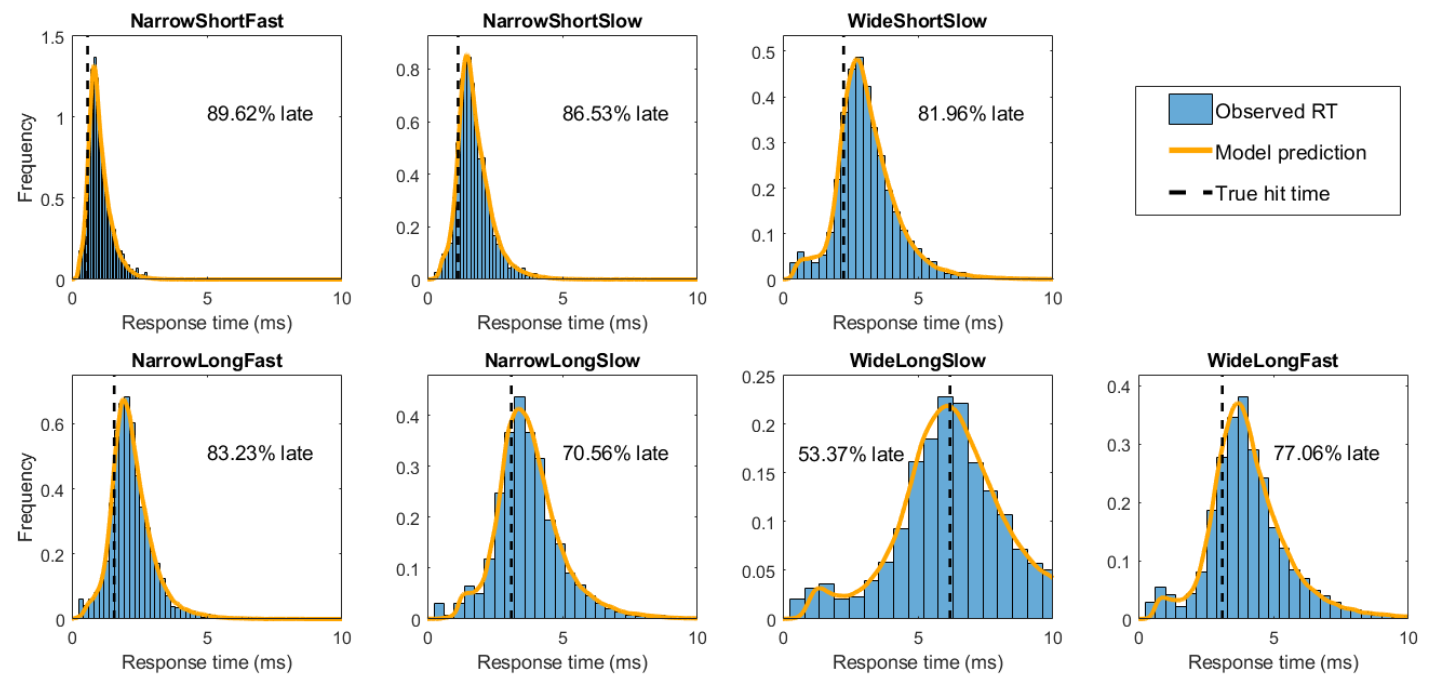

\section{Figure 4}

Distribution of response times for each condition (blue histograms), along with the response time distributions predicted by the model (yellow lines). Dashed black lines indicate the point at which the ball actually crossed the far edge of the occlusion, i.e., when responses should ideally have been made. As we noted above, all WideShortFast trials presented a WideShortSlow stimulus, so there are no WideShortFast condition results to show.

Essentially, this was true for each individual condition as well as the aggregate response times, although longer true arrival times (long distance, slow movement conditions) tended to decrease the number of late response times. As we show in the model-based analysis, lateness is also alleviated by a greater exposure to the ball prior to its occlusion. This resulted in the WideLongSlow condition (bottom row, middleright of Figure 4) having the fewest tardy responses.

\section{Model-based analysis}

To evaluate the cognitive processes underlying performance on the anticipatory timing task, we used the shifted Wald accumulator model described above (Balc1 \& Simen, 2016; Anders et al., 2016). This model typically has four parameters per condition: the drift rate $\gamma$, the diffusion rate $\sigma$, the threshold $\theta$, and non-decision time / shift parameter $\tau$. Ordinarily, the diffusion parameter of this model is fixed to $\sigma=.1$ or 1 to set the scale of the model; however, this is not required and other parameters may be fixed or vary across conditions (Donkin et al., 2009). In this study, we are more interested in how uncertainty - as well as how the values of drift and diffusion relative to the threshold - change with time. Therefore, instead of fixing the diffusion rate, we fixed the threshold to $\theta=1$. This reflects the observation that participants knew the location of right side of the occlusion - which did not vary across conditions - and therefore could fix the criterion they used to terminate the decision process by always using the same point of reference.

To evaluate how our manipulations (occlusion length, travel distance, and ball speed) affected uncertainty on the task, we used effects coding to linearly predict drift rate, diffusion rate, and non-decision time in each condition, as a function of each of the three manipulations. The baseline condition was the narrow occlusion, short distance, and slow ball movement condition (NarrowShortSlow). Main effects of making the occlusion wider, making the distance longer, or making the ball faster were quantified with linear additive effects. We also estimated interactions between conditions, but none of these interactions showed any credible effects, so we present only the main effects of each manipulation. These main effects, along with the grand mean / NarrowShortSlow condition, are shown in Table 1.

If the Wald model sufficiently accounts for our manipulations, we should expect the drift rate (tracking the passage of time while the ball was occluded) and non-decision time (tracking the length of time the ball was visible) to correspond to the true length of time the ball took to cross the screen. Thus, they should respond to each of the manipulations according to how they affected the length of time the stimulus was visible / occluded: drift should be highest with Short, Narrow, and Fast conditions, while non-decision time should be highest with Long, Narrow, and Slow conditions (Wide occlusions decrease the visible time and rate at which the ball passes through the occlusion as a percentage of its width). If participants are otherwise unresponsive to our manipulations and/or performing perfectly, then we should find no effects of any manipulations on the accumulation of uncertainty ( $\sigma$ estimates). 


\section{Table 1}

Summary of group-level parameter estimates for the effectscoded Wald accumulator model for Study 1. Each of the parameters was estimated on a log scale to ensure that their values would be positive. The parameter values for an individual condition can be computed by taking the exponential of the linear combination of the coefficient for the baseline condition (NarrowShortSlow, denoted 0) and the relevant coefficients for the condition of interest. For example, the diffusion rate for NarrowShortFast would be $\exp \left(\sigma_{0}+\sigma_{\text {Fast }}\right)$. All effects are credibly nonzero, so none are displayed in bold as elsewhere in the text.

\begin{tabular}{|c|c|c|c|}
\hline & Parameter & Mean & $95 \%$ HDI \\
\hline \multirow{4}{*}{ 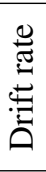 } & $\gamma_{0}$ & -1.01 & {$[-1.09,-0.89]$} \\
\hline & $\gamma_{\text {Wide }}$ & -0.16 & {$[-0.18,-0.15]$} \\
\hline & $\gamma_{\text {Long }}$ & -0.40 & {$[-0.41,-0.38]$} \\
\hline & $\gamma_{\text {Fast }}$ & 0.32 & {$[0.31,0.34]$} \\
\hline \multirow{4}{*}{ 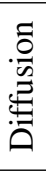 } & $\sigma_{0}$ & -2.12 & {$[-2.26,-1.95]$} \\
\hline & $\sigma_{W i d e}$ & 0.04 & {$[-0.00,0.06]$} \\
\hline & $\sigma_{\text {Long }}$ & -0.27 & {$[-0.30,-0.24]$} \\
\hline & $\sigma_{\text {Fast }}$ & 0.28 & {$[0.25,0.31]$} \\
\hline \multirow{4}{*}{ 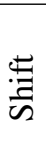 } & $\tau_{0}$ & 0.43 & {$[0.36,0.51]$} \\
\hline & $\tau_{\text {Wide }}$ & -0.29 & {$[-0.34,-0.27]$} \\
\hline & $\tau_{\text {Long }}$ & 0.09 & {$[0.07,0.11]$} \\
\hline & $\tau_{\text {Fast }}$ & -0.25 & {$[-0.27-0.24]$} \\
\hline
\end{tabular}

As indicated by the parameter estimates, participants accurately perceived that the ball would arrive sooner when the distance to travel was short (negative effect of $\gamma_{\text {Long }}$ ) or when it was moving quickly (positive $\gamma_{\text {Fast }}$ ). They also accurately believed that the ball would take longer to arrive when it was occluded for longer (negative $\gamma_{\text {Wide }}$ ), indicating that drift was specifically indexing representations of uncertainty in the ball's location when it was occluded as opposed to when it was still visible. This suggests that the timer mechanism was used to track the passage of time and the ball's motion only when there was uncertainty about the relationship between the two. The degree to which drift changed under uncertainty was strongly related to general difficulties with timing (see self-report results below) and is particularly significant to understanding patterns of lateness, so we examine this effect in greater detail in the Discussion.

As for the diffusion parameter quantifying uncertainty, we found that reducing the length of time participants were able to see the ball - either by reducing the travel distance (Short), or increasing the ball speed (Fast) - increased how uncertain they became about its position (increased $\sigma$ ). The longer participants were able to see the ball the better they were able to assess its velocity, which reduced uncertainty about its position while it was occluded. While the HDI of the effect of occlusion length (Wide) was smaller and marginally crossed zero, it also appeared to increase the value of $\sigma$, fur- ther suggesting that the degree of uncertainty participants experienced was closely to related to the length of time they had to assess its motion prior to occlusion.

Finally, the non-decision time corresponded closely to the length of time the ball was visible. Wider occlusion and faster ball speed led to a smaller visible time, and thus negative effects of of $\tau_{\text {Wide }}$ and $\tau_{\text {Fast }}$. Conversely, a longer distance led to a slightly longer visible time: the occlusion took the same absolute size on the screen in Long/Short conditions, and therefore constituted a smaller fraction of the total width of the screen in the Long condition. This led to a slightly positive effect of $\tau_{\text {Long }}$, corresponding to a greater visible time in the Long relative to Short condition.

This model provided an excellent overall account of response times on the timing task, as shown by the yellow lines in Figure 4. There were no major discrepancies with the observed response times (histograms) on the aggregate or individual levels, suggesting that the Wald model provides a good account of behavior on both anticipatory and reactive timing tasks.

One outstanding question related to the non-decision time and drift rate estimates is how closely they align with the optimal values. As opposed to whether they simply track the time when the stimulus was visible, we can examine their values relative to the true length of time the ball was visible or occluded. To do so, we subtracted non-decision time estimates from the length of time the ball was visible, and multiplied the drift rates by the length of the time the ball was actually behind the occlusion. Consequently, we were able to assess whether participants under- or over-estimated the ball's movement, as it moved across the screen and behind the occlusion. We index these corrected estimates as $\gamma^{*}$ for drift rates and $\tau^{*}$ for non-decision time.

When corrected for the true duration for which the ball remained occluded, drift rates in each condition were only half as high as they should be to accurately track the passage of time: $\left.\mathrm{M}\left(\gamma^{*}\right)=0.50,95 \% \mathrm{HDI}=[0.30,0.83]\right)$. However, this varied across conditions, with Wide / Slow conditions having drift rates substantially closer to 1 (e.g., Wide/Short/Slow $\left.\mathrm{M}\left(\gamma_{W S S}^{*}\right)=0.84\right)$ than Narrow / Fast conditions (e.g., Narrow/Long/Fast $\left.\mathrm{M}\left(\gamma_{N L F}^{*}\right)=0.30\right)$.

Interestingly, non-decision time contributes almost nothing to the tardiness of participants' responses. If participants were simply taking a long time to execute their motor responses, we would expect longer non-decision times relative to the true length of time the stimulus was visible. However, non-decision times were calibrated almost perfectly to the true duration of the stimulus: $\mathrm{M}\left(\tau^{*}\right)=-0.01$ (95\% HDI $=$ $[-0.31,0.46])$. This suggests that participants were tracking the motion of the ball as it was visible, and preparing their responses for when they expected it to arrive, with a high degree of precision and accuracy. As a result, the lateness of their judgments appears to be driven entirely by faulty repre- 
sentations of (changes in) the stimulus' position while it was occluded. We investigate this hypothesis in greater depth in Study 2.

\section{Self-report measures}

Since the parameters of the timing model were estimated hierarchically, we can evaluate not only the group-level trends as above, but also the individual-level fits. Specifically, for each of the parameters in Table 1, we have a parameter for each person in the study that can be used as an individual difference in how they represented the passage of time under uncertainty. These individual differences were then used to predict high-level timing problems, including procrastination and our novel timing problems scale. First, it is worth summarizing performance on each of the scales. For procrastination (Steel, 2010), participants' average score was 28.39 ( $S D=7.79$ ). Reliability, assessed using Cronbach's $\alpha$, was $\rho_{T}=0.89$. Scores on our new timing problems scale were calculated by summing the responses (strongly disagree $=1$ to strongly agree $=5$ ) after reverse coding. Participants had an average score of $46.80(S D=10.09)$. It also showed a high degree of reliability, with Cronbach's $\alpha$ coefficient $\rho_{T}$ $=0.85$. Scores on each of the scales were closely related to one another $(r=.72,95 \% \mathrm{HDI}=[0.52,0.93])$, indicating (perhaps unsurprisingly) that participants who struggled with procrastination also struggled with timeliness and forethought.

As for model parameters, both procrastination and timing problem scales were related to drift rates, which index how well participants were able to track the passage of time under uncertainty. The correlations between these self-report scales and drift rates of the Wald model are shown in Table 2. In general, participants who had generally low base drift rates (low $\gamma_{0}$ ) had more problems with timing, indicating that lowlevel perceptual problems with tracking the passage of time propagate to substantial problems with timing in common real-world scenarios. Furthermore, timing problems were exacerbated by an inability to represent the passage of time under uncertainty, indicated by the relationship between $\gamma_{\text {Wide }}$ (our primary manipulation of stimulus uncertainty).

Put together, these correlations appear to indicate that problems with tardiness and forward planning stem from an inability to accurately track and anticipate the passage of time, particularly when there are no visual cues to its passage. Specifically, the degree to which participants became more uncertain about the passage of time when the ball was occluded translated to difficulty with accomplishing everyday tasks where monitoring time is integral to success. We suggest that this is likely a result of subjective deflation of the passage of time, where time appears to participants to pass more slowly than it objectively does. This leads to late estimates of when the ball should arrive at the far end of the occlusion, creating the patterns of results we se in Figure 4.
Table 2

Estimated correlation coefficients for the relationship between drift rate parameters $(\gamma)$ and scores on the timing problems and procrastination scales in Study 1. Numbers in brackets correspond to 95\% HDIs on the relationship between model parameters and scale scores. Those HDIs excluding zero are shown in bold.

\begin{tabular}{c|cc} 
Parameter & Timing & Procrastination \\
\hline$\gamma_{0}$ & $\mathbf{- 0 . 2 9}[-.51,-.07]$ & $-0.11[-.33, .11]$ \\
$\gamma_{\text {Wide }}$ & $\mathbf{- 0 . 3 2}[-.54,-.11]$ & $\mathbf{- 0 . 3 5}[-.55,-.11]$ \\
$\gamma_{\text {Long }}$ & $-0.20[-.41, .02]$ & $\mathbf{- 0 . 3 0}[-.54,-.08]$ \\
$\gamma_{\text {Fast }}$ & $0.24[-.01, .44]$ & $\mathbf{0 . 2 5}[.04, .47]$
\end{tabular}

Procrastination was similarly related to drift rates and thus performance on the task, although participants who were high on procrastination did not necessarily share the generalized problems with tracking the passage of time $\left(\gamma_{0}\right)$ that participants with timing problems did. Instead, participants who struggled with procrastination had greater difficulty dealing with manipulations of the time it took the stimulus to cross the screen, such as the ball movement speed $\left(\gamma_{\text {Fast }}\right)$ and the distance it had to travel $\left(\gamma_{\text {Long }}\right)$. As a result, procrastination appeared to be more closely related to temporal distance, while tardiness and timing problems are more closely related to tracking the passage of time under uncertainty.

\section{Study 2}

An important finding from Study 1 is that participants appeared to track the ball as moving as little as half as fast as it actually moved through the occlusion (estimates of $\gamma$, corrected for the true occluded duration, ranging from around .4-.8). A possible explanation could be that subjects used the ball's motion when it was visible to estimate the length of time they would have to wait once it was occluded. Adopting such a strategy would circumvent the need to actually represent the motion of the ball once it moved behind the occlusion, as participants could simply enter their responses after a fixed delay determined by the characteristics of the stimulus. The difficulties people encountered on the task could therefore potentially be attributed to how well they assessed the duration of the waiting period, as opposed to how well they actively tracked the passage of time.

To address this possibility, Study 2 asked participants to actively track the location of the ball by prompting them to respond with its position at specific points in time. This allowed us to evaluate how far participants were ahead or behind its true motion as well as how this representation changed over time.

The conditions in Study 1 (Narrow/Wide, Short/Long, Slow/Fast) were further subdivided into four location conditions, which varied in terms of where the ball was when participants were prompted for its location. This yielded a 
total of 32 unique combinations of condition and ball position. On each trial, the position of a participant's click designating where they thought the center of the ball was was transformed into a number between 0 and 1 , indicating what proportion of the occlusion they believed the ball had traversed. Therefore, the variability in responses around the ball location is normalized by the size of the occlusion. As a result, the same variance in responses on the scale of the screen (true variance) will be a greater proportion of the narrow occlusion, and thus the baseline variance of responses in narrow conditions will be higher.

As in Study 1, we used effects coding to estimate the impact of each of the manipulations. Due to multiple measurements within each condition (four different timings of the prompt), we not only predicted the mean (drift) and variance (diffusion) of responses in each condition, but also how the mean and variance changed as the ball moved further into the occlusion. For instance, it could be the case that a wide occlusion would result in similar uncertainty about the ball's position at early time points compared to a narrow occlusion, but that uncertainty in the ball's position would grow more steeply as the ball moved along behind a wide relative to a narrow occlusion. We therefore predicted the mean and variance of the ball's position as a linear function of the point at which it was prompted (i.e., .2, .4, .6 or .8 of the distance through the occlusion).

Formally, the ball's position at time $t, P(t)$, was predicted as a truncated normal distribution:

$$
P(t) \sim N\left(\mu_{0}+\mu \cdot t, \sigma_{0}^{2}+\sigma^{2} \cdot t\right) T(0,1) .
$$

As mentioned in the introduction (Figure 1, Equation 1), the position of a Wald accumulator must be normally distributed. Furthermore, the mean and variance of the normally distributed state must increase linearly with time; hence the linear functions of time for $t$ in Equation 2. We allow the intercept and slope terms to vary with the condition manipulations:

$$
\begin{aligned}
\mu_{0} & =m_{0}+m_{\text {Wide }} \cdot W+m_{\text {Long }} \cdot L+m_{\text {Fast }} \cdot F \\
\mu & =\gamma_{0}+\gamma_{\text {Wide }} \cdot W+\gamma_{\text {Long }} \cdot L+\gamma_{\text {Fast }} \cdot F \\
\sigma_{0}^{2} & =\exp \left(s_{0}+s_{\text {Wide }} \cdot W+s_{\text {Long }} \cdot L+s_{\text {Fast }} \cdot F\right) \\
\sigma^{2} & =\exp \left(v_{0}+v_{\text {Wide }} \cdot W+v_{\text {Long }} \cdot L+v_{\text {Fast }} \cdot F\right)
\end{aligned}
$$

The variables $\mathrm{W}, \mathrm{L}$, and $\mathrm{F}$ are indicators / dummy effect codes for wide occlusion, long travel distance, and fast ball movement manipulations, where 0 represents narrow, short, and slow conditions and 1 represents wide, long, and fast conditions. In total, this gives us 16 parameters ( 4 baseline mean, 4 drift, 4 baseline variance, and 4 diffusion rates) to account for distributions of responses across all 32 conditions.

As in Study 1, these parameters were estimated using a hierarchical Bayesian approach with uninformative priors. The JAGS model code is available on the OSF page (osf.io/b28sq). Overall, the model did a good job of accounting for the distributions of responses in each condition, on both the individual and group levels. The aggregate fits for each of the conditions and time points are shown in Figure 5. There were a few individuals who responded near the left edge of the occlusion on every trial, who show up as "lumps" on the left side of each of these distributions. Because the model was fit hierarchically, there was some shrinkage in its predictions that prevented capturing these outliers. This is typically desirable (Kruschke, 2014; Kruschke \& Liddell, 2018; Boehm et al., 2018), although sometimes it can overconstrain true yet extreme parameter values (Scheibehenne et al., 2013). In this case, the model ignoring extreme responses reflects the likely scenario that these responses are actually outliers, produced by participants who were not fully engaged in the task. However, since $a$ priori data cleaning procedures were not established to specifically eliminate them, we leave such responses in for further analyses.

The group-level estimates and HDIs of the model are shown in Table 3. In general, participants had a slight bias to report the ball as having moved further than it actually had for early prompts, as indicated by the positive intercept for the ball position $m_{0}>0$. As a result, participants overestimated the position of the ball at early time points. This is shown in Figure 6: when the ball had traversed only the first $20 \%$ of the occlusion, participants tended to overestimate its position.

This overestimation was quickly countered by a low drift rate $\gamma_{0}$. In ideal circumstances, this rate should be approximately 0.20 - the true length of the occlusion that the ball traversed between each time point that could be prompted. However, as shown in Table 3, the true drift was typically much lower at around 0.04 to 0.08 . This fairly dramatic effect could be driven in part by floor and ceiling effects - participants could not respond below 0 or above 1 , and so low responses may be biased upward and high responses biased downward. However, this appears insufficient to explain the effects. Each model we tested - including a truncated normal model (reported here) and Markov random walk models with reflecting or absorbing boundaries ${ }^{1}$ - found extremely sim-

\footnotetext{
${ }^{1}$ In these models, ball location was represented as a random walk in a 101-state system. Its initial state was assumed to be at state 1 (left edge of the occlusion) and its motion was represented using an intensity matrix constructed with drift and diffusion parameters (see Kvam et al., 2015, 2021). To control for boundary effects, we tried a version of the model where the far edge was a reflecting boundary (all responses entering state 101 went to state 100 ) or where it was an an absorbing boundary (all entries in state 101 stayed there). We fit the model by comparing the proportion of the evidence in each state against the number of responses there, for each response time and condition. However, neither model fit better (when comparing DIC values) than the truncated normal model reported in the main text. Furthermore, each model showed an attenuated drift rate (much lower than 0.20), indicating that the effects are
} 
Table 3

Summary of group-level parameter estimates (mean, 95\% HDIs) for the effects-coded truncated normal distribution model for Study 2. Parameters represent participants' mean estimates of the distance the ball had traveled (Location $\mu)$ and the log variance of these estimates $\left(\log \left(\sigma^{2}\right)\right)$.

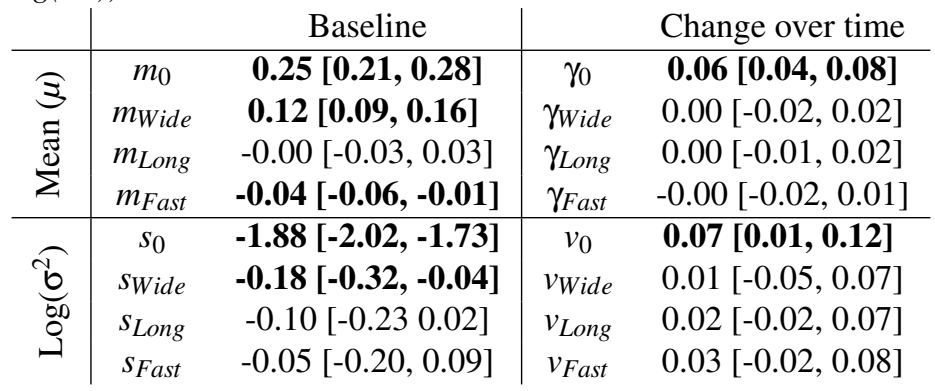
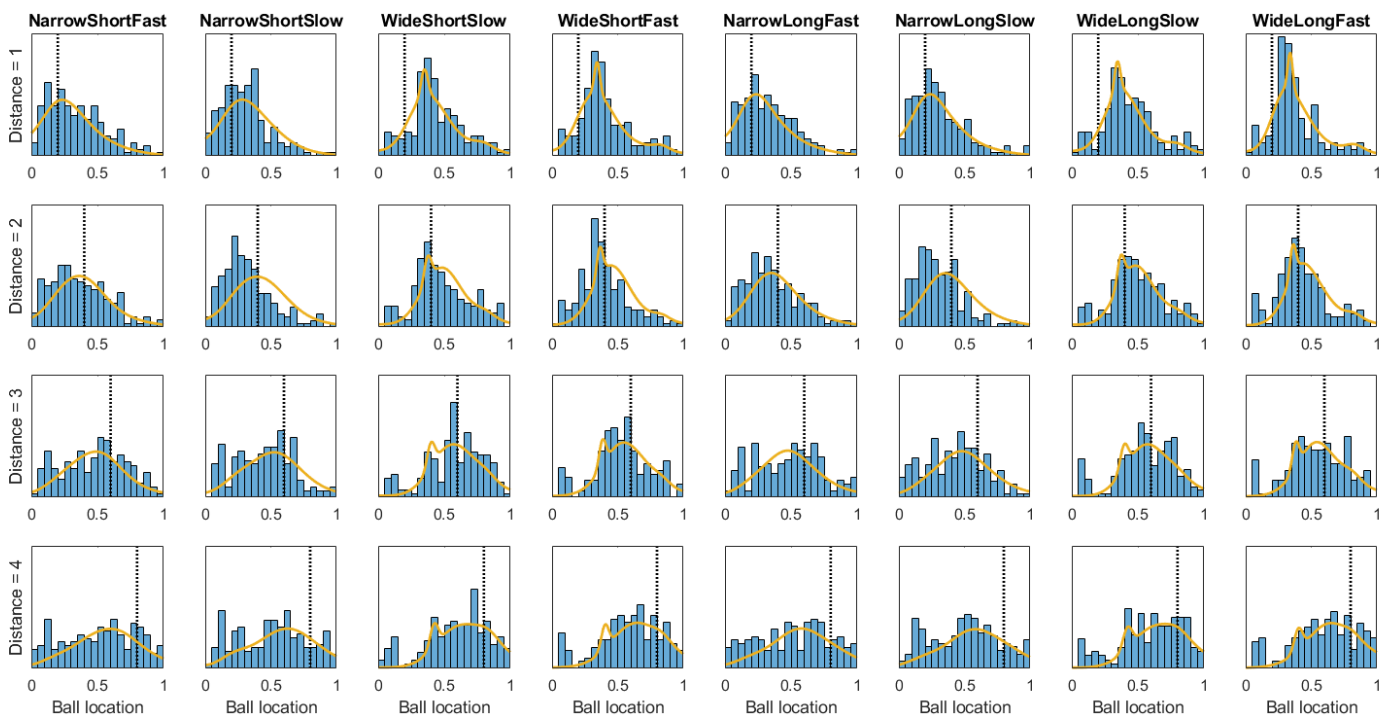

Figure 5

Distributions of responses from participants in Study 2 (blue histograms) for each condition (columns) and ball location (rows). The true location of the ball in each panel is shown as a dashed vertical black line, and the model predictions derived from an aggregate of the best fits to each participant-are shown as overlaid yellow lines.

ilar drift rates despite multiple different approaches to correcting or accounting for boundary effects.

In contrast to Study 1, there were few credible effects of the condition manipulations on either biases in location responses $m$ or drift rates $\gamma$. The only manipulation that changed the apparent position of the ball within the occlusion is the Narrow/Wide manipulation, where a wider occlusion led participants to perceive the ball as having moved slightly further than it actually had $\left(m_{\text {Wide }}>0\right)$. By contrast, manipulations of Long/Short and Fast/Slow had small or negligible effects on the mean of ball location responses. Essentially, the mean positions of participants' responses were sensitive to the position of the ball $\left(\gamma_{0}>0\right)$ but less than it ought to be $\left(\gamma_{0}<1\right)$, and this effect was consistent across conditions.

In addition to the mean responses for ball locations, we also examined how the variance of these responses changed over time. According to the model, the variance in responses should grow linearly with time. This prediction was confirmed the data $\left(v_{0}>0\right.$ in Table 3$)$, and is visually clear in the patterns of variance shown in Figure 7. As with the drift rates, the increase in variance over time was largely unaffected by condition manipulations, evidenced by the other $v$ parameters being nearly centered at zero.

The intercept of the variance of responses was nonzero, and can be computed as the exponential of the $s_{0}$ estimates: $M\left(\exp \left(s_{0}\right)\right)=0.15(95 \%$ HDI $=[0.14,0.19])$. This simply indicates that not all variance in responses was due to the

unlikely to be attributable to floor or ceiling effects, as the Markov models would have accounted for these. 

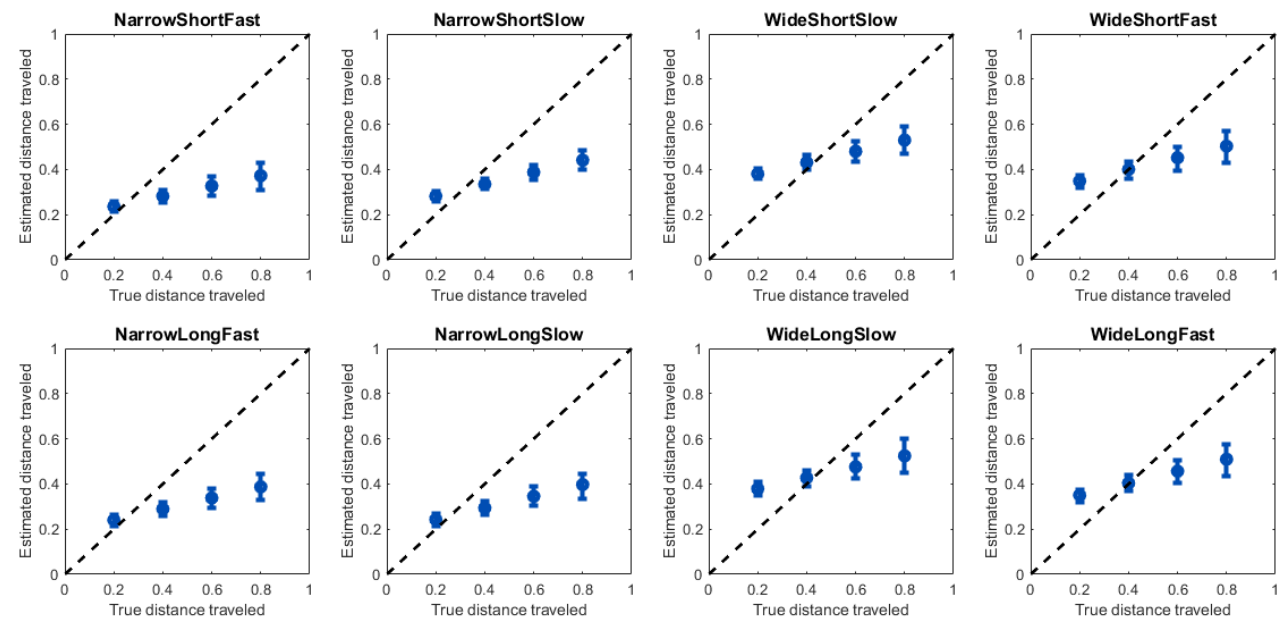

Figure 6

Symbols show mean responses in each of the conditions of Study 2. Error bars indicate the 95\% HDIs on the group-level means, computed from the posterior parameter estimates provided in Table 3.
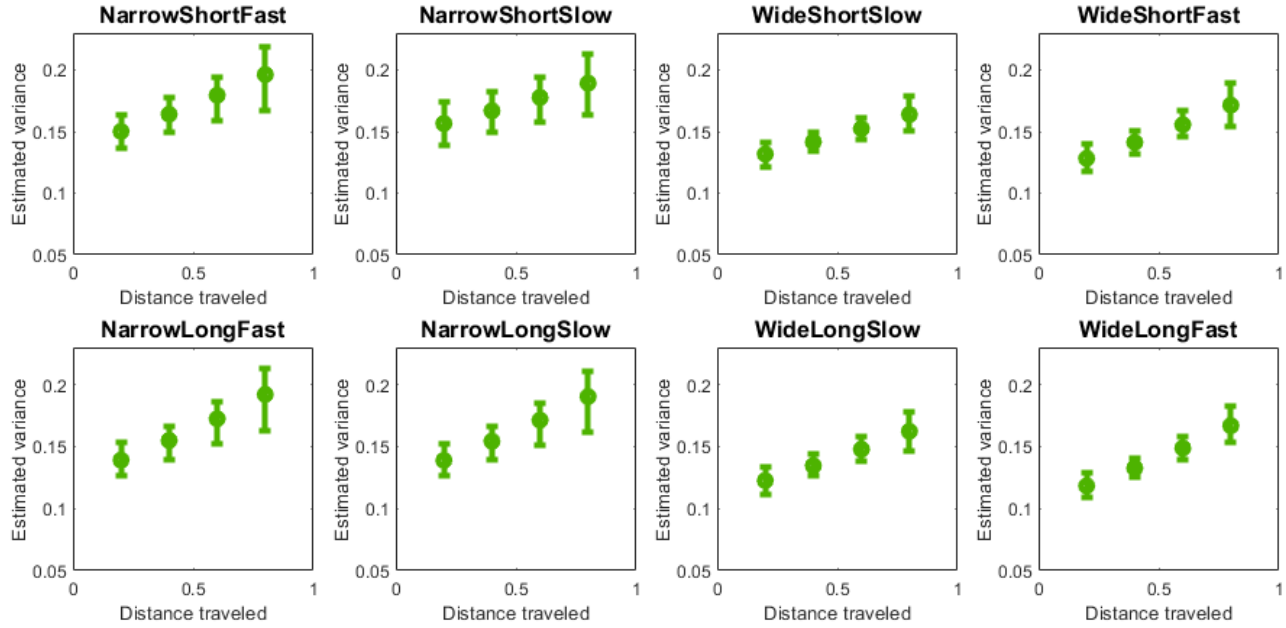

Figure 7

Symbols show response variance (averaged across participants) in each of the conditions of Study 2. Error bars indicate the $95 \%$ HDIs on the group-level means of the model variance parameters, computed from the posterior parameter estimates provided in Table 3.

motion of the stimulus, but rather to motor error or to starting point variability (Ratcliff et al., 2018). As we might expect, having a wide occlusion decreased this baseline variability - because the size of the rectangle itself gets larger when a wide occlusion is used rather than a narrow one, the same variability on a raw scale (pixels) gets smaller as a proportion of the total size of the box (divided by width of occlusion).

\section{Discussion}

The results of our studies are broadly supportive of the Wald accumulator model as a cognitive mechanism underly- ing anticipatory timing. The distributions of response times in Study 1 closely followed a Wald distribution in each condition, and the distributions of responses in Study 2 followed a truncated normal distribution. Furthermore, the variance of the response distributions grew linearly with time (Figure 7 ), which is a core prediction of the model. As a result, the effects-coded models were able to account for behavior on the tasks (Figures 4 and 5) as well as quantify how participants responded to manipulations of the stimulus and uncertainty in its location.

Notably, both studies uncovered drift rates that were substantially less than 1 (Tables 1 and 3), indicating that par- 
ticipants fell behind in tracking the movement of the ball once it was occluded. This resulted in the majority of responses being significantly late in Study 1, and resulted in estimations of the ball's location that fell behind over time in Study 2. It is perhaps no coincidence that the degree of attenuation in the drift rates relative to the objective motion of the stimulus aligns with the "filled-duration" illusion, where time periods with no stimulus in them are perceived to be shorter than those with a stimulus present (Wearden et al., 2007). Although the traditional filled-duration illusion occurs with auditory stimuli (Thomas \& Brown, 1974), it is found to be similar (Buffardi, 1971) or even accentuated in visual and tactile domains (Williams et al., 2019). In each of these cases, time periods with no new sensory input or with a smaller number of items in sensory buffers are experienced subjectively as approximately $60 \%$ of the duration of matched time periods where a more arousing stimulus is present (Wearden et al., 2007).

Moreover, a filled-duration illusion appears to explain why participants responded too late in most conditions. If each person perceives the duration of the "occluded" time as shorter than the equivalent duration of "visible" time, then they must wait longer - in objective and absolute time - to make the occluded time equal to the visible one. For instance, we can imagine a case where the ball is visible for exactly half of its trajectory and where filled durations are seen as twice as long as unfilled ones. If the ball is visible for 2 seconds, then a participant would need to wait 4 seconds when it is occluded to attempt to match the subjective durations of occluded and visible time periods.

If we attribute this effect to the pacemaker mechanism of an internal clock (Wing \& Kristofferson, 1973b,a), then we should expect the pacemaker to run at approximately 55-65\% of its rate when the ball is occluded relative to when it is visible (Wearden et al., 2007). It should therefore come as no surprise that drift rates were around this range, averaging $\gamma \approx .50$ in Study 1 and $\gamma \approx .31$ in Study $2 .^{2}$ The effects in our studies appeared to be even more extreme than those in the literature, as they dissociated the "baseline" time that participants assumed had passed (intercept for ball position / nondecision time) from direct estimates of their representations of the passage of time as the trial unfolded. This suggests that the filled duration illusion may even be an under-estimate of the true effect, due to participants assuming a minimum amount of time has passed (intercept), but actually tracking the passage of time during unfilled durations as lasting 30$50 \%$ of the unfilled durations.

Despite the explanatory power of a filled-duration illusion, the results cannot be understood without the effects of uncertainty. Specifically, if the filled-duration illusion alone were responsible for the results, we would not have expected faster or more on-time / early responses when the ball was occluded (Figure 4). In the Wald model, this effect emerges from greater uncertainty in the ball's location - variance in the accumulator specified by $\sigma$ that grows over time. Just as increases in drift can lead to faster responses, so can increases in variance / diffusion (Ratcliff \& Rouder, 1998). Ironically, participants' higher levels of uncertainty in longocclusion / slow-movement conditions led to their response times growing closer to the correct ball arrival time. This is not a prediction that would be implied by the filled-duration illusion - which would predict that distorted perceptions of time would result in more (not fewer) late responses for the longer occlusion durations - but it is a natural consequence of the dynamic accumulation models we implemented to account for behavior on the tasks. The Wald timing model approach, and specifically accounting for how our manipulations influenced dynamic uncertainty $(\sigma)$, is therefore critical to understanding participants' performance and their representations of timing in anticipatory tasks.

\section{Manipulations \& timing problems}

Another illuminating finding from our studies is the connection between low-level perceptual time inflation - such as the magnitude of the filled-duration effect in $\gamma$ - and highlevel problems with planning, lateness, and procrastination. Individual differences in the ability to perceive the passage of time - even in the relatively short-duration and simplified timing task that our participants engaged in - would have to result from fundamental mechanisms (such as a universal pacemaker) that pervade many aspects of behavior. Our results suggest that people who struggle with timing in their daily lives may in fact be simply unable to accurately track the passage of time. Specifically, this appears to stem from difficulty in assessing "unfilled" spans of time where there are no referents for its passage (like watching a clock) or novel stimuli. The growth of uncertainty during these unfilled periods may not be entirely detrimental - as it can result in more on-time actions - but overall it creates lower and lower precision in the representation of time as it passes. People who struggle with time management and tracking may benefit particularly from visual or auditory cues that allow them to accurately track time (Ahrens \& Sahani, 2011), as these sensory inputs provide anchors that pacemaker timing mechanisms can leverage to produce higher-fidelity representations of duration.

Timing difficulty also appears to be exacerbated by manipulations of uncertainty, with the Narrow/Wide manipulation being particular hard for participants who had timing and procrastination problems (Table 2). Learning the relationship between ball speed and the rate at which a timer must be set is a challenging problem, and individual differences in learning are sure to propagate from low-level deficits to high-level

\footnotetext{
${ }^{2}$ The value of $\gamma_{0}$ from Table 3 must be multiplied by 5 , as the estimate is relative to the distance it traverses per time point (.20) rather than the total distance it traverses over a trial.
} 
prediction errors in the absence of feedback (Moore et al., 1998). Differences in task structure leading to differences in apparent timing ability are certainly not unheard of, and it may be that these participants benefit more from direct experience and feedback allowing them to better make anticipatory timing decisions (Wearden, 2003). Certainly, future interventions incorporating feedback could evaluate whether participants who have problems with timing or procrastination are able to improve over time as they practice anticipatory timing under uncertainty.

One way the task structure appears to have influenced our results is in differences in the effect of condition manipulations on drift / diffusion parameters $(\gamma / \sigma$ in Study 1 and $\gamma /$ $v$ in Study 2, respectively). Each manipulation had relatively large and ubiquitous effects on these dynamic processes in Study 1 , yet entirely absent in Study 2. It could be that the difference in task - tracking the location of a hidden object versus predicting its time of arrival - is driving differences in timing strategies between the two studies. Tracking the motion of an object (Study 2), as opposed to the duration it will be occluded (Study 1), may simply be less malleable by manipulations of speed, uncertainty, or distance. One potential way to look at this would be to examine eye movements during the task, and identifying whether participants who "follow" the motion of the occluded ball (as incentivized in Study 2) are less susceptible to manipulations of uncertainty, distance, or ball speed.

\section{Implications \& predictions}

The most important predictions from the diffusion-based models of timing - including both the TopDDM (Balc1 \& Simen, 2016) and TRDM (Hawkins \& Heathcote, 2021) were verified in the experimental results. Specifically, response times in Study 1 followed a Wald distribution closely (Figure 4), and both the mean and variance of position estimates increased linearly with time in Study 2 (Figures $6 \& 7$ ). Despite this, there are a few elements of the results that serve as words of caution and potentially as modifications to both models. The dramatic reduction of drift rates under uncertainty signify conditions under which the timer mechanism may not function optimally in either model. As this effect increases with uncertainty manipulations where less is happening on-screen, it appears to be related to the filled-duration illusion. The perception of the passage of time, and thus the timer mechanism in the TopDDM / TRDM, is likely to be skewed by the presence of a dynamic stimulus. Therefore, we might expect timer drifts to be lower when a stimulus is static (such as face / word recognition memory paradigms Ratcliff, 1978b) relative to expanded judgment paradigms where the stimulus changes from moment to moment (Smith \& Vickers, 1989; Ratcliff \& Smith, 2010).

If we accept that the drift rate of the timer is affected by static / dynamic stimuli due to the filled duration illusion, it becomes clear that the type of stimulus will have an effect on response time distributions under time pressure. Specifically, decisions should be terminated more quickly when the stimulus changes over time, because the dynamic features of the stimulus allow participants to better track the passage of time. As a result, response times under time pressure should be more right-skewed for static stimuli then for dynamic ones. This could potentially explain why some studies have found support for collapsing boundary models (Malhotra et al., 2018; Hawkins et al., 2012, 2015) while others have not (Voskuilen et al., 2016). The rate at which a decision is terminated varies as a function of the stimulus itself, meaning that the timer will carry greater influence in some paradigms (specifically, dynamic stimuli) than others.

This prediction is not limited to timer-based models of early decision termination, either. Regardless of how a decision is terminated, the brain requires some mechanism to track the passage of time. If all timer mechanisms are susceptible to temporal distortion induced by the filled-duration illusion, then there is no reason to believe that timers subserving the collapse of thresholds are immune to these manipulations. Timers underlying the rate of threshold collapse, urgency signals, and termination of choice should all be affected. As a result, the optimality of choices based on collapsing threshold or timer mechanisms may be contingent on the stimulus presented. The optimality of collapsingboundary approaches to decision-making may therefore be compromised by our inability to track the passage of time, which is determined by the type of stimulus (dynamic ones that fill an interval, static ones that do not, or some combination of the two). Such effects may be a particular problem for internal timing on preferential choice problems (Tajima et al., 2016; Bhui, 2019; Busemeyer et al., 2019), where nearly all stimuli are static and there are few cues to the passage of time.

\section{Conclusion}

The ability to keep track of time is critical to triggering decisions in both decisions under time pressure and anticipatory timing. Understanding how the passage of time is represented, or in this case misrepresented under particular conditions, allows us to diagnose the cognitive processes that are responsible for patterns of difficulty with high-level planning and timing tasks. As we showed, grappling with uncertainty in low-level perceptual decisions propagates to problems with tardiness, planning ahead, and starting and completing tasks on time. Furthermore, beyond anticipatory timing, these cognitive mechanisms also carry implications for decision models that invoke timer mechanisms to control choice. Put together, the studies we present here suggest that people have systematic biases in time representation that can be attributed to subjective inflation of time under uncertainty. Evident in both anticipatory response times and projections 
about stimulus changes, individual differences in ability to respond to manipulations of uncertainty are key to understanding how people respond to and control the dynamics of their environment.

\section{Acknowledgments}

This work was supported by a University of Florida Informatics Institute Graduate Fellowship awarded to K.S. This research was partially supported by an Australian Research Council (ARC) Discovery Project (DP180103613) to G.H.

\section{References}

Ahrens, M. B., \& Sahani, M. (2011). Observers exploit stochastic models of sensory change to help judge the passage of time. Current Biology, 21(3), 200-206.

Algom, D., Eidels, A., Hawkins, R. X., Jefferson, B., \& Townsend, J. T. (2015). Features of response times: Identification of cognitive mechanisms through mathematical modeling. In J. R. Busemeyer, Z. Wang, J. T. Townsend, \& A. Eidels (Eds.), The oxford handbook of computational and mathematical psychology (p. 63--98). Oxford University Press.

Anders, R., Alario, F., \& Van Maanen, L. (2016). The shifted wald distribution for response time data analysis. Psychological methods, 21(3), 309-.

Balc1, F., \& Simen, P. (2016). A decision model of timing. Current opinion in behavioral sciences, 8, 94-101.

Balota, D. A., \& Yap, M. (2011). Moving beyond the mean in studies of mental chronometry the power of response time distributional analyses. Current Directions in Psychological Science, 20, 160-166.

Bareš, M., Lungu, O. V., Husárová, I., \& Gescheidt, T. (2010). Predictive motor timing performance dissociates between early diseases of the cerebellum and parkinson's disease. The Cerebellum, 9(1), 124-135.

Bhui, R. (2019). Testing optimal timing in value-linked decision making. Computational Brain \& Behavior, 2(2), 85-94.

Boehm, U., Marsman, M., Matzke, D., \& Wagenmakers, E.J. (2018). On the importance of avoiding shortcuts in applying cognitive models to hierarchical data. Behavior research methods, 50(4), 1614-1631.

Bogacz, R., Brown, E., Moehlis, J., Holmes, P., \& Cohen, J. D. (2006). The physics of optimal decision making: A formal analysis of models of performance in twoalternative forced-choice tasks. Psychological Review, 113(4), 700-765. doi: 10.1037/0033-295X.113.4.700
Bogacz, R., Hu, P. T., Holmes, P. J., \& Cohen, J. D. (2010). Do humans produce the speed-accuracy trade-off that maximizes reward rate? The Quarterly Journal of Experimental Psychology, 63(5), 863-891.

Bowman, N. E., Kording, K. P., \& Gottfried, J. A. (2012). Temporal integration of olfactory perceptual evidence in human orbitofrontal cortex. Neuron, 75(5), 916-927. doi: 10.1016/j.neuron.2012.06.035

Buffardi, L. (1971). Factors affecting the filled-duration illusion in the auditory, tactual, and visual modalities. Perception \& Psychophysics, 10(4), 292-294.

Busemeyer, J. R., Gluth, S., Rieskamp, J., \& Turner, B. M. (2019). Cognitive and neural bases of multi-attribute, multi-alternative, value-based decisions. Trends in Cognitive Sciences, 23(3), 251-263.

Cacioppo, J. T., \& Petty, R. E. (1982). The need for cognition. Journal of Personality and Social Psychology, 42(1), 116-131.

Carroll, C. A., O'donnell, B. F., Shekhar, A., \& Hetrick, W. P. (2009). Timing dysfunctions in schizophrenia as measured by a repetitive finger tapping task. Brain and Cognition, 71(3), 345-353.

Casini, L., Pech-Georgel, C., \& Ziegler, J. C. (2018). It's about time: revisiting temporal processing deficits in dyslexia. Developmental Science, 21(2), e12530.

Churchland, A. K., Kiani, R., \& Shadlen, M. N. (2008). Decision-making with multiple alternatives. Nature neuroscience, 11(6), 693-702.

De Leeuw, J. R. (2015). jspsych: A javascript library for creating behavioral experiments in a web browser. Behavior research methods, 47(1), 1-12.

de Leeuw, J. R., \& Motz, B. A. (2016). Psychophysics in a web browser? comparing response times collected with javascript and psychophysics toolbox in a visual search task. Behavior Research Methods, 48(1), 1-12.

Donkin, C., Brown, S. D., \& Heathcote, A. (2009). The overconstraint of response time models: Rethinking the scaling problem. Psychonomic Bulletin \& Review, 16(6), 1129-1135. doi: 10.3758/PBR.16.6.1129

Drugowitsch, J., Moreno-Bote, R., Churchland, A. K., Shadlen, M. N., \& Pouget, A. (2012). The cost of accumulating evidence in perceptual decision making. The Journal of Neuroscience, 32(11), 3612-3628. doi: 10.1523/ JNEUROSCI.4010-11.2012 
Edland, A., \& Svenson, O. (1993). Judgment and decision making under time pressure. In Time pressure and stress in human judgment and decision making (pp. 2740). Springer.

Eriksen, B. A., \& Eriksen, C. W. (1974). Effects of noise letters upon the identification of a target letter in a nonsearch task. Perception \& psychophysics, 16(1), 143-149.

Fautrelle, L., Mareschal, D., French, R., Addyman, C., \& Thomas, E. (2015). Motor activity improves temporal expectancy. PLoS One, 10(3), e0119187.

Haines, N., Kvam, P. D., Irving, L. H., Smith, C., Beauchaine, T. P., Pitt, M. A., ... Turner, B. (2020). Learning from the reliability paradox: How theoretically informed generative models can advance the social, behavioral, and brain sciences. PsyArXiv, psyarxiv.com/xr7y3.

Hawkins, G. E., Brown, S. D., Steyvers, M., \& Wagenmakers, E.-J. (2012). An optimal adjustment procedure to minimize experiment time in decisions with multiple alternatives. Psychonomic bulletin \& review, 19(2), 339-348.

Hawkins, G. E., Forstmann, B. U., Wagenmakers, E.-J., Ratcliff, R., \& Brown, S. D. (2015). Revisiting the evidence for collapsing boundaries and urgency signals in perceptual decision-making. The Journal of Neuroscience, 35(6), 2476-2484.

Hawkins, G. E., \& Heathcote, A. (2021). Racing against the clock: Evidence-based versus time-based decisions. Psychological Review, 128(2), 222-263.

Heathcote, A., Popiel, S. J., \& Mewhort, D. (1991). Analysis of response time distributions: An example using the stroop task. Psychological bulletin, 109(2), 340.

Heitz, R. P. (2014). The speed-accuracy tradeoff: history, physiology, methodology, and behavior. Frontiers in $\mathrm{Neu}$ roscience, $8,150$.

Hilbig, B. E. (2016). Reaction time effects in lab-versus web-based research: Experimental evidence. Behavior Research Methods, 48(4), 1718-1724.

Hitchcock, P., Fried, E. I., \& Frank, M. (2021). Computational psychiatry needs time and context. Annual Review of Psychology.

Hoffer, A., \& Osmond, H. (1962). The relationship between mood and time perception. Psychiatric Quarterly Supplement.

Ivry, R. B. (1996). The representation of temporal information in perception and motor control. Current opinion in neurobiology, 6(6), 851-857.
Jones, C. R., \& Jahanshahi, M. (2014). Motor and perceptual timing in parkinson's disease. Neurobiology of Interval Timing, 265-290.

Jones, C. R. G., Claassen, D., Yu, M., Spies, J. R., Malone, T., Dirnberger, G., ... Kubovy, M. (2011). Modeling accuracy and variability of motor timing in treated and untreated parkinson's disease and healthy controls. Frontiers in Integrative Neuroscience, 5, 81.

Keele, S. W., \& Hawkins, H. L. (1982). > explorations of individual differences relevant to high level skill. Journal of Motor Behavior, 14(1), 3-23.

Kim, E., \& McAuley, J. D. (2013). Effects of pitch distance and likelihood on the perceived duration of deviant auditory events. Attention, Perception, \& Psychophysics, 75(7), 1547-1558.

Kruschke, J. K. (2014). Doing bayesian data analysis: A tutorial with R, JAGS, and STAN. Academic Press.

Kruschke, J. K., \& Liddell, T. M. (2018). The bayesian new statistics: Hypothesis testing, estimation, meta-analysis, and power analysis from a bayesian perspective. Psychonomic Bulletin \& Review, 25(1), 178-206.

Kvam, P. D., Busemeyer, J. R., \& Pleskac, T. J. (2021). Temporal oscillations in preference strength provide evidence for an open system model of constructed preference. Scientific reports, $11(1), 1-15$.

Kvam, P. D., Pleskac, T. J., Yu, S., \& Busemeyer, J. R. (2015). Interference effects of choice on confidence: Quantum characteristics of evidence accumulation. Proceedings of the National Academy of Sciences, 112(34), 10645-10650. doi: 10.1073/pnas.1500688112

Lee, M. D., \& Wagenmakers, E.-J. (2014). Bayesian cognitive modeling: A practical course. Cambridge university press.

Lemke, M. R., Koethe, N. H., \& Schleidt, M. (1999). Timing of movements in depressed patients and healthy controls. Journal of affective disorders, 56(2-3), 209-214.

Lerche, V., \& Voss, A. (2020). When accuracy rates and mean response times lead to false conclusions: A simulation study based on the diffusion model. The Quantitative Methods for Psychology, 16(2), 107-119.

Luce, R. D. (1986). Response times (No. 8). Oxford University Press.

MacLeod, C. M. (1992). The stroop task: The" gold standard" of attentional measures. Journal of Experimental Psychology: General, 121(1), 12. 
Malhotra, G., Leslie, D. S., Ludwig, C. J., \& Bogacz, R. (2018). Time-varying decision boundaries: insights from optimality analysis. Psychonomic bulletin \& review, 25(3), 971-996.

Martino, D., Hartmann, A., Pelosin, E., Lagravinese, G., Delorme, C., Worbe, Y., \& Avanzino, L. (2019). Motor timing in tourette syndrome: the effect of movement lateralization and bimanual coordination. Frontiers in neurology, 10,385 .

Meegan, D. V., Aslin, R. N., \& Jacobs, R. A. (2000). Motor timing learned without motor training. Nature neuroscience, 3(9), 860-862.

Michon, J. A. (1967). Timing in temporal tracking. Institute for Perception RVO-TNO Soesterberg, The Netherlands.

Miletić, S., \& van Maanen, L. (2019). Caution in decisionmaking under time pressure is mediated by timing ability. Cognitive Psychology, 110, 16-29.

Moore, J. W., Choi, J.-S., \& Brunzell, D. H. (1998). Predictive timing under temporal uncertainty: the time derivative model of the conditioned response. In D. A. R. . C. E. Collyer (Ed.), Timing of behavior: Neural, psychological, and computational perspectives (p. 3--34). The MIT Press.

O’Donnell, B. F., Hetrick, W. P., Vohs, J. L., Krishnan, G. P., Carroll, C. A., \& Shekhar, A. (2004). Neural synchronization deficits to auditory stimulation in bipolar disorder. Neuroreport, 15(8), 1369-1372.

Pariyadath, V., \& Eagleman, D. (2007). The effect of predictability on subjective duration. PloS one, 2(11), e1264.

Ratcliff, R. (1978a). A theory of memory retrieval. Psychological Review, 85(2), 59-108.

Ratcliff, R. (1978b). A theory of memory retrieval. Psychological Review, 85(2), 59-108. doi: 10.1037/0033-295X 85.2.59

Ratcliff, R., \& McKoon, G. (2008). The diffusion decision model: Theory and data for two-choice decision tasks. Neural Computation, 20(4), 873-922. doi: 10.1162/ neco.2008.12-06-420

Ratcliff, R., \& Rouder, J. N. (1998). Modeling response times for two-choice decisions. Psychological Science, 9(5), 347-356. doi: 10.1111/1467-9280.00067

Ratcliff, R., \& Smith, P. L. (2010). Perceptual discrimination in static and dynamic noise: The temporal relation between perceptual encoding and decision making. Journal of Experimental Psychology: General, 139(1), 70.
Ratcliff, R., Smith, P. L., Brown, S. D., \& McKoon, G. (2016). Diffusion decision model: Current issues and history. Trends in Cognitive Sciences, 20(4), 260-281.

Ratcliff, R., Voskuilen, C., \& McKoon, G. (2018). Internal and external sources of variability in perceptual decisionmaking. Psychological Review, 125(1), 33-46.

Reed, A. V. (1973). Speed-accuracy trade-off in recognition memory. Science, 181(4099), 574-576.

Reimers, S., \& Stewart, N. (2015). Presentation and response timing accuracy in adobe flash and html5/javascript web experiments. Behavior research methods, 47(2), 309327.

Reynolds, A., Garton, R., Kvam, P. D., Griffin, V., Sauer, J., Osth, A., \& Heathcote, A. (2021). A dynamic model of deciding not to choose. Journal of Experimental Psychology: General, 150, 42-66.

Reynolds, A., Kvam, P. D., Osth, A. F., \& Heathcote, A. (2020). Correlated racing evidence accumulator models. Journal of Mathematical Psychology, 96, 102331.

Scheibehenne, B., Rieskamp, J., \& Wagenmakers, E.-J. (2013). Testing adaptive toolbox models: A Bayesian hierarchical approach. Psychological Review, 120(1), 39-64. doi: $10.1037 / \mathrm{a} 0030777$

Shiffrin, R., Lee, M., Kim, W., \& Wagenmakers, E.-J. (2008). A survey of model evaluation approaches with a tutorial on hierarchical bayesian methods. Cognitive Science: A Multidisciplinary Journal, 32(8), 1248-1284. doi: 10.1080/03640210802414826

Simen, P., Balci, F., deSouza, L., Cohen, J. D., \& Holmes, P. (2011). A model of interval timing by neural integration. Journal of Neuroscience, 31(25), 9238-9253.

Simen, P., Vlasov, K., \& Papadakis, S. (2016). Scale (in) variance in a unified diffusion model of decision making and timing. Psychological Review, 123(2), 151.

Smith, P. L. (2010). From poisson shot noise to the integrated Ornstein-Uhlenbeck process: Neurally principled models of information accumulation in decision-making and response time. Journal of Mathematical Psychology, 54(2), 266-283.

Smith, P. L. (2016). Diffusion theory of decision making in continuous report. Psychological Review, 123(4), 425451. doi: 10.1037/rev0000023

Smith, P. L., \& Vickers, D. (1989). Modeling evidence accumulation with partial loss in expanded judgment. Journal of Experimental Psychology: Human Perception and Performance, 15(4), 797. 
Steel, P. (2010). Arousal, avoidant and decisional procrastinators: Do they exist? Personality and Individual Differences, 48(8), 926-934.

Tajima, S., Drugowitsch, J., \& Pouget, A. (2016). Optimal policy for value-based decision-making. Nature communications, 7(1), 1-12.

Thomas, E. C., \& Brown, I. (1974). Time perception and the filled-duration illusion. Perception \& Psychophysics, 16(3), 449-458.

Tse, P. U., Intriligator, J., Rivest, J., \& Cavanagh, P. (2004). Attention and the subjective expansion of time. Perception \& psychophysics, 66(7), 1171-1189.

Ulrich, R., Nitschke, J., \& Rammsayer, T. (2006). Perceived duration of expected and unexpected stimuli. Psychological research, 70(2), 77-87.

Vickers, D. (1970). Evidence for an accumulator model of psychophysical discrimination. Ergonomics, 13(1), 3758.

Voskuilen, C., Ratcliff, R., \& Smith, P. L. (2016). Comparing fixed and collapsing boundary versions of the diffusion model. Journal of Mathematical Psychology, 73, 59-79. doi: 10.1016/j.jmp.2016.04.008

Wald, A., \& Wolfowitz, J. (1949). Bayes solutions of sequential decision problems. Proceedings of the National Academy of Sciences of the United States of America, 35(2), 99-102.

Wearden, J. H. (2003). Applying the scalar timing model to human time psychology: Progress and challenges. Time and mind II: Information processing perspectives, 21-39.

Wearden, J. H., Norton, R., Martin, S., \& Montford-Bebb, O. (2007). Internal clock processes and the filled-duration illusion. Journal of Experimental Psychology: Human Perception and Performance, 33(3), 716.

Wickelgren, W. A. (1977). Speed-accuracy tradeoff and information processing dynamics. Acta Psychologica, 41(1), 67-85. doi: 10.1016/0001-6918(77)90012-9

Williams, E. A., Yüksel, E. M., Stewart, A. J., \& Jones, L. A. (2019). Modality differences in timing and the filledduration illusion: Testing the pacemaker rate explanation. Attention, Perception, \& Psychophysics, 81(3), 823-845.

Williamson, J. R., Quatieri, T. F., Helfer, B. S., Ciccarelli, G., \& Mehta, D. D. (2014). Vocal and facial biomarkers of depression based on motor incoordination and timing. In Proceedings of the 4th international workshop on audio/visual emotion challenge (pp. 65-72).
Wing, A. M., \& Kristofferson, A. (1973b). The timing of interresponse intervals. Perception \& Psychophysics, 13(3), 455-460.

Wing, A. M., \& Kristofferson, A. B. (1973a). Response delays and the timing of discrete motor responses. Perception \& Psychophysics, 14(1), 5-12.

Wittmann, M., \& Lehnhoff, S. (2005). Age effects in perception of time. Psychological reports, 97(3), 921-935.

Wittmann, M., Leland, D. S., Churan, J., \& Paulus, M. P. (2007). Impaired time perception and motor timing in stimulant-dependent subjects. Drug and alcohol dependence, 90(2-3), 183-192.

Wolff, P. H. (2002). Timing precision and rhythm in developmental dyslexia. Reading and Writing, 15(1), 179-206.

Zelaznik, H. N., Vaughn, A. J., Green, J. T., Smith, A. L., Hoza, B., \& Linnea, K. (2012). Motor timing deficits in children with attention-deficit/hyperactivity disorder. $\mathrm{Hu}$ man movement science, 31(1), 255-265.

Ziessler, M., \& Nattkemper, D. (2011). The temporal dynamics of effect anticipation in course of action planning. Quarterly Journal of Experimental Psychology, 64(7), 1305-1326.

\section{Appendix A: Timing problems scale}

The individual questions that were presented on the timing problems scale are shown in Table S1. Responses were coded from 1 (strongly disagree) to 5 (strongly agree), with questions 1, 3, 4, 9, 11, 12, 13, and 14 reverse coded. Question 8 was dropped, as it could be interpreted either as prospective time management strategy (something I do because I am good at time management) or as a compensatory strategy (I plan a few extra minutes because I know I am bad at time management). Scored responses to the remaining 16 items were summed to get an overall summary score for the scale for each participant. 
KONSTANTINA SOKRATOUS

Table S1

Self-report timing scale used to measure problems with time management and lateness.

\begin{tabular}{|c|c|c|c|c|c|}
\hline & $\begin{array}{l}\text { Strongly } \\
\text { disagree }\end{array}$ & $\begin{array}{c}\text { Somewhat } \\
\text { disagree }\end{array}$ & $\begin{array}{l}\text { Neither agree } \\
\text { nor disagree }\end{array}$ & $\begin{array}{l}\text { Somewhat } \\
\text { agree }\end{array}$ & $\begin{array}{l}\text { Strongly } \\
\text { agree }\end{array}$ \\
\hline 1. I like to plan ahead. & $\bigcirc$ & 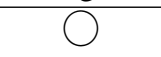 & 0 & & O \\
\hline 2. I often feel rushed. & 0 & & 0 & & O \\
\hline 3. I feel well-prepared for tasks I must & $\bigcirc$ & & & & D \\
\hline 4. I plan out my day. & 0 & & & & \\
\hline $\begin{array}{l}\text { 5. I often find myself needing to play } \\
\text { "catch-up" on work during my free } \\
\text { time. }\end{array}$ & $\bigcirc$ & & & & ) \\
\hline $\begin{array}{l}\text { 6. I am frequently late to events I plan } \\
\text { to attend. }\end{array}$ & O & & & & \\
\hline $\begin{array}{l}\text { 7. It feels like there is not enough time } \\
\text { in the day to accomplish my goals. }\end{array}$ & O & & & & \\
\hline $\begin{array}{l}\text { 8. I often plan a few minutes of } \\
\text { "buffer" time into my schedule. }\end{array}$ & O & & O & & \\
\hline 9. I often feel over-prepared. & & & & & \\
\hline $\begin{array}{l}\text { 10. When traveling, I arrive at my } \\
\text { destination later than I had planned. }\end{array}$ & 0 & & & & \\
\hline 11. I usually plan out my week. & & & & & \\
\hline $\begin{array}{l}\text { 12. It feels like I have more than } \\
\text { enough time to achieve my daily } \\
\text { goals. }\end{array}$ & O & & & & \\
\hline $\begin{array}{l}\text { 13. I typically arrive early to events I } \\
\text { plan to attend. }\end{array}$ & 0 & & $\Omega$ & & \\
\hline 14. I usually plan out my month. & O & & O & & \\
\hline $\begin{array}{l}\text { 15. I sometimes under-estimate how } \\
\text { long tasks will take. }\end{array}$ & 0 & & & & \\
\hline $\begin{array}{l}\text { 16. I wish that I were better at plan- } \\
\text { ning for the future. }\end{array}$ & 0 & $\Omega$ & O & O & \\
\hline $\begin{array}{l}\text { 17. I often find myself struggling to } \\
\text { keep pace with the tasks I need to ac- } \\
\text { complish during the day. }\end{array}$ & O & O & O & O & $\bigcirc$ \\
\hline
\end{tabular}

